\title{
Mental illness and cardiovascular mortality: searching for the links
}

\section{David S. Goldbloom, Paul Kurdyak}

$\infty \quad$ See related article, page 779

I $\mathrm{n}$ the Canadian health care system, where universal access is promoted as a goal (if not a reality), we need to understand where and why such access is unequal or inequitable - the latter term implying elements of unjustness and unfairness beyond simple differences in quantity. In this issue of $C M A J$, Kisely and colleagues ${ }^{1}$ report on an administrative database approach to addressing access to invasive cardiovascular and neurovascular procedures as well as rates of death from circulatory disease among patients in Nova Scotia with psychiatric disorders.

Nova Scotia has a long tradition of excellence in psychiatric epidemiology. ${ }^{2}$ The authors' sophisticated methodological approach may send some readers back to their epidemiology and biostatistics primers, but such complexity was necessary to order and interpret data from the thousands of people studied.

The prevalence of psychiatric disorders, from this database, was $15 \%$, resembling that of other provinces. People who had had contact with health services for psychiatric problems accounted for $16 \%$ of the deaths from circulatory disease (2839/I7 665) over the 7-year period studied; the agestandardized mortality-rate ratio was significantly elevated: I.3I. Of the patients who had received invasive vascular procedures, I5\% overall (I685/II 2I8) fit the study definition of psychiatric patients, but subanalyses revealed a decreased likelihood of receiving procedures among those patients admitted to hospital for psychiatric illness or treated by psychiatrists (as opposed to primary care physicians) as outpatients.

These findings are consistent with 2 previous reports ${ }^{3,4}$ of decreased likelihood of cardiac procedures after myocardial infarction among US patients with psychiatric disorders. Interestingly, other studies have documented a striking increase in the use of health services after cardiac-related admissions to hospital by patients with depression compared with those without this disorder. ${ }^{5,6}$ It may be that the patients who are less likely to receive cardiac procedures are those with mental illnesses that are more severe and chronic in nature.

The implication of Kisely and colleagues' findings is that the difference in rates of receipt of procedures may be a contributor to increased deaths from cardiovascular causes among people with psychiatric disorders. However, to attrib$n$ ute an increase in deaths to a reduction in the use of cardiovascular procedures requires, at the very least, the opportunity to adjust for cardiovascular risk factors. The use of administrative data does not permit risk-factor measurement or adjustment. In patients with psychiatric disorders, the impact of cardiovascular risk factors is likely to be substantial. We already know that the presence of a concurrent psychiatric disorder, such as depression, increases rates of death from myocardial infarction. ${ }^{7}$ In addition, among people with schizophrenia, the mean Io-year risk of coronary heart disease or stroke, based on Framingham predictor variables, is significantly elevated compared with that of the general population. ${ }^{8,9}$

We also know that cigarette smoking is far more common among people with a psychiatric disorder than in the general population. Indeed, patients with schizophrenia have rates of smoking as high as $88 \%$, with even higher rates among those admitted to hospital for schizophrenia than among outpatients. Among people with a diagnosis of depression or bipolar disorder, smoking rates may be as high as $60 \%$, compared with less than $25 \%$ in the general population. ${ }^{10}$ Moreover, efforts to quit smoking in these patient groups are less successful than in the population at large. ${ }^{10}$ We know as well that psychotropic medications have a variety of metabolic and cardiac effects that may be additive to other factors that increase the risk of death. ${ }^{9}$ Finally, we also know that obesity, an additional risk factor, is more prevalent among people who have a psychiatric disorder. ${ }^{11}$ This database study cannot tell us the relative contribution of reduced frequencies of procedures per se to the increase in deaths.

\section{These measurable in- equalities challenge our notion of universality.}

Kisely and colleagues have revealed an inequality in the likelihood of receiving secondary prevention interventions for cardiovascular illness among people with psychiatric disorders. The huge burden of cardiovascular risk factors in this subpopulation suggests a similar, if not more striking, inequality in primary prevention. The reasons for such inequity (and the reasons underlying the higher rates of death) are undoubtedly complicated. Speculatively, they may include decreased access to specialty cardiac care, discrimination against people with a psychiatric disorder, decreased compliance with general medical care by people with psychiatric disorders (particularly among those who have paranoia), decreased consent rates for proposed invasive procedures, lifestyle factors (e.g., homelessness), socioeconomic factors or other as-yet-unidentified variables. 
Note that this database study documents the receipt of procedures, which is not exactly the same as access to them. We cannot know from this study whether people with a psychiatric disorder are able to visit physicians who would make the clinical decision to recommend an invasive procedure, for example, nor how often such recommendations are accepted. Unequal access would suggest that, given the same clinical indications for an invasive vascular procedure in 2 patients, the one who also has a psychiatric disorder is less likely to obtain it. Inequitable access would suggest that this difference is not only measurable but also, in the context of the values of Canada's health care system and society, unfair.

Kisely and colleagues' data obligates us to look more closely at the entire sequence, from risk factors for circulatory disease through its symptoms, assessment and management, as well as responses (illness, disability and death) to interventions, whether lifestyle/behavioural, pharmacological or invasive. Broadly, this scrutiny needs to include the attitudes of both patients and physicians toward the physical health needs of people with psychiatric disorders. The barriers to ensuring equity in health care for people with chronic psychiatric illnesses pose a serious challenge to our notion of universality.

\section{This article has been peer reviewed.}

David Goldbloom is Senior Medical Advisor with Education and Public Affairs, Centre for Addiction and Mental Health, and Professor, Department of Psychiatry, University of Toronto; Paul Kurdyak is Head of Emergency/Crisis Services and Research, General Psychiatry Program, Centre for Addiction and Mental Health, and Lecturer, Department of Psychiatry, University of Toronto, Toronto, Ont.
Competing interests: None declared.

Contributors: David Goldbloom and Paul Kurdyak collaborated in drafting and revising this article, and approved the final version for publication.

\section{REFERENCES}

I. Kisely S, Smith M, Lawrence D, et al. Inequitable access for mentally ill patients to some medically necessary procedures. CMAJ 2007;176(6):779-84.

2. Tremblay MA. Alexander H. Leighton's and Jane Murphy's scientific contributions to psychiatric epidemiology: a personal appreciation. Transcult Psychiatry 2006; 43:7-20.

3. Druss BG, Bradford DW, Rosenheck RA, et al. Mental disorders and use of cardiovascular procedures after myocardial infarction. JAMA 2000;283:506-II.

4. Young JK, Foster DA. Cardiovascular procedures in patients with mental disorders. JAMA 2000;283:3198-9.

5. Frasure-Smith N, Lesperance F, Gravel G, et al. Depression and health-care costs during the first year following myocardial infarction. J Psychosom Res 2000;48: $47 \mathrm{I}-8$.

6. Sullivan M, Simon G, Spertus J, et al. Depression-related costs in heart failure care. Arch Intern Med 2002;162:1860-6.

7. Van Melle JP, De Jonge P, Spijkerman TA, et al. Prognostive association of depression following myocardial infarction with mortality and cardiovascular events: a meta-analysis. Psychosom Med 2004; 66:8I4-22.

8. McReadie RG; Scottish Schizophrenia Lifestyle Group. Diet, smoking and cardiovascular risk in people with schizophrenia: descriptive study. BrJ Psychiatry 2003; I83:534-9.

9. Goff DC, Sullivan LM, McEvoy JP, et al. A comparison of ten-year cardiac risk estimates in schizophrenia patients from the CATIE study and matched controls. Schizophr Res 2005;80:45-53.

Io. Kalman D, Morisette SB, George TP. Co-morbidity of smoking and patients with psychiatric and substance use disorders. Am J Addict 2005; I4: I06-23.

II. Newcomer JW. Medical risk in patients with bipolar disorder and schizophrenia. JClin Psychiatry 2006;67(suppl 9):25-30.

Correspondence to: Dr. David Goldbloom, Centre for Addiction and Mental Health, 250 College St., Toronto ON M5T IR8; david_goldbloom@camh.net

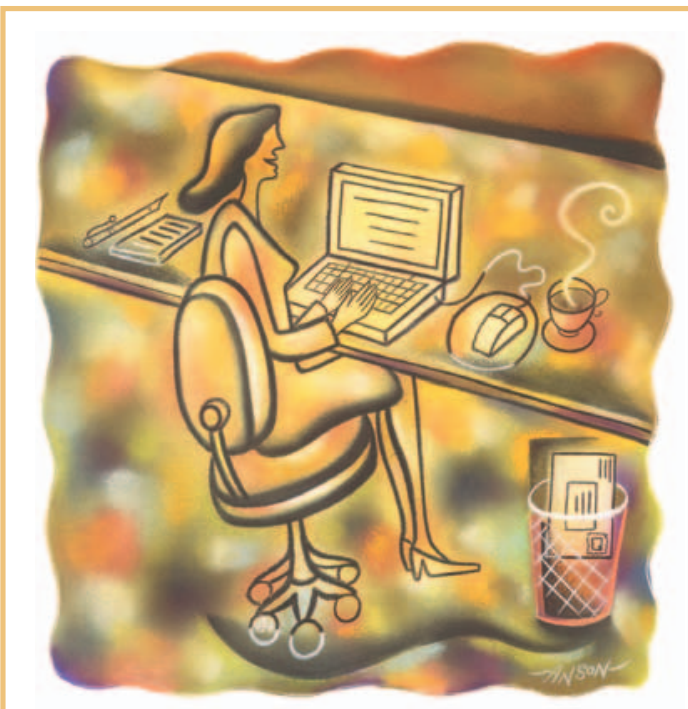

\title{
Online manuscript submissions and peer review
}

\author{
NOW AVAILABLE AT CMAJ \\ http://mc.manuscriptcentral.com/cmaj
}

